\title{
A Comparative Study of Shear-Wave Elastography and Strain Elastography on a Breast Phantom for Diagnosis of Tumor and Cyst
}

\author{
Mahdi Al-Qahtani ${ }^{1}$, Eraj Humayun Mirza ${ }^{2}$, Mubarak Dhafer Al-Qahtani ${ }^{3}$ and \\ Mohammed Fahad Al-Muqati ${ }^{4}$ \\ Department of Biomedical Technology, College of Applied Medical Sciences, King Saud University, \\ Riyadh, KSA \\ 1amahdi@ksu.edu.sa, 2emirza@ksu.edu.sa, ${ }^{3}$ m.z_20@hotmail.com, ${ }^{4}$ king123-@hotmail.com
}

\begin{abstract}
A lump in breast may suggest a possible development of breast cancer. As the tumor develops, it changes the physical property of the tissue by changing its stiffness. To detect the tumor generally mammography is performed. The findings of mammography are often inaccurate as it does not take into account the stiffness of tissue. Elastography is an imaging technique to measure the stiffness of tissues. In the current study, we have used a breast phantom to analyze differences between a tumor and a cyst via comparing shear-wave elastography and strain elastography techniques. Both the techniques performed equally for the diagnosis of tumour. However, it is suggested that strain elastography must be used in the diagnosis of a cyst as it provides enhanced details of the surrounding tissues.
\end{abstract}

Key-words: Elastogrpahy, Shear-wave, Strain, Ultrasound, breast, tumor, phantom.

\section{Introduction}

Changes in elasticity of tissue is attributed to pathological condition [1]. Various cancers appear as hard nodules as a result of increased density, while other diseases involves deposition of fat or collagen that might alter the tissue elasticity. Cysts filled with fluid may also be invisible to traditional ultrasound examination. In several cases, a diminished pathological lesion or a lesion that is not superficial may not be detected via conventional ultrasound technique [2]. Conventional ultrasound is a qualitative method [3], however there was a need to a more quantitative approach to differentiate healthy tissues from the diseased. To overcome this issue ultrasound elastography was introduced.

Elastography is a medical imaging technique that is non-invasive, which detects tumors according to their stiffness when compared to normal tissue.

Elastography is a non-invasive medical imaging technique. The purpose of this technique is detecting tumors based on their stiffness (elasticity) compared to normal tissue. Ultrasonic imaging used for the most common type of elastography to compare the shapes of the tissue under examination before and after it is compressed slightly. Normal tissue tend to be less stiff cancerous tumors. Elastogram is an image show as different shades of light and dark, also has different degree of stiffness. The elastogram show up 
Mahdi Al-Qahtani, Eraj Humayun Mirza, Mubarak Dhafer Al-Qahtani and Mohammed Fahad Al-Muqati; A Comparative Study of

Shear-Wave Elastography and Strain Elastography on a Breast Phantom for Diagnosis of Tumor and Cyst. Journal of Biomedical Engineering and Medical Imaging, Volume 2, No 3, June (2015) , pp 24-28

many tumors, including breast tumors better than conventional ultrasonic images. Elastography is an imaging modality that apply a small axial uniform compression on the tissue that provides insight into the elastic properties of biological tissue [4]. Currently two basic types of elastography is performed; the shear wave elastography (SWE) and strain elastography (SE) [5]. SWE uses a set of shear waves to measure the modulus of tissue and provides tissue stiffness based on pressure values determined by the machine [6, 7]. On the other hand SE make use of application of localized strain manually by the operator and determining the relative strain of a lesion in comparison to surrounding tissues [8, 9]. Recent studies have demonstrated similar use of both SWE and SE techniques individually for various tissues. However, their comparable results are not studied significantly. Here we report the evaluation of breast tumor in a phantom using both the SWE and SE techniques.

\section{Materials \& Methods}

\subsection{Elastography examination}

SWE and SE was performed on elastography breast phantom. Breast tumor and cyst was identified using linear transducer with a bandwidth ranging from 4-15 $\mathrm{MHz}$ for SWE while 5-15 MHz linear transducer was used for SE. After determining the tumor and cyst via B-mode ultrasound the SWE and SE elastography images were acquired and most crisp image was selected from a pool of several images. No compression was applied when performing SWE imaging, however in case of SE imaging a light compression was applied which was confirmed by the feedback loop in the machine itself.

This study was performed in accordance with guidelines followed by ethical review board of King Saud University (KSU). SE procedures were performed with SonixTouch Q+ from Ultrasonix Medical Corporation, 130-4311 Viking Way, Richmond, Canada, at Department of biomedical technology, College of applied Medical Sciences, KSU. SWE procedures were performed with Aixplorer from SuperSonic Imagine Les Jardins de la Duranne, Aix-en-Provence, France, at department of radiology, King Fahad Medical City. Elastography breast phantom was purchased from CAE healthcare USA, 6300 Edgelake Drive Sarasota, FL, USA.

\subsection{Image evaluation}

After the selection of appropriate image from the pool of several images from SWE and SE examination, the lesion size and area of tumor and cyst were recorded. Tissue stiffness for SWE was recorded as displayed in the machine. Results for SWE displayed blue coloration in the range of $30 \mathrm{kPa}$ while the red color demonstrated tissue stiffness to be around $180 \mathrm{kPa}$. In case of SE, the strain ratio between normal and pathological tissue was measured. Blue coloration showing a soft tissue while red coloration demonstrating a hard tissue.

\section{Results \& Discussion}

The images obtained from breast phantom using two different elastography machines were analyzed using Tsukuba elasticity score patterns [10]. It was observed that both the elastography equipment displayed similar images (figure 1B, 1D \& 2B, 2D) in B-mode which was effective in differentiating a tumor from cyst. Furthermore it was observed that elastography patterns varied from equipment to equipment. A significant variation was observed among the color patterns of the tumor (figure $1 A \& 2 A$ ). The strain ratios were only measureable in SonixTouch $Q+$ ultrasound machine. The strain ratio was 0.17 for the 
tumor observed. Furthermore, color patterns of cyst were also different. Aixplorer from Supersonic, depends on shear wave technology so it displays the cyst as cavity while all its surrounding tissue as normal tissue (figure 2A) whereas, on the other hand, the SonixTouch $Q+$ from Analogic depends on external compression, so it provides elastography images based on relative stiffness. It displays cyst in blue color as soft tissue while surrounding tissue as hard (red) in figure $2 \mathrm{C}$

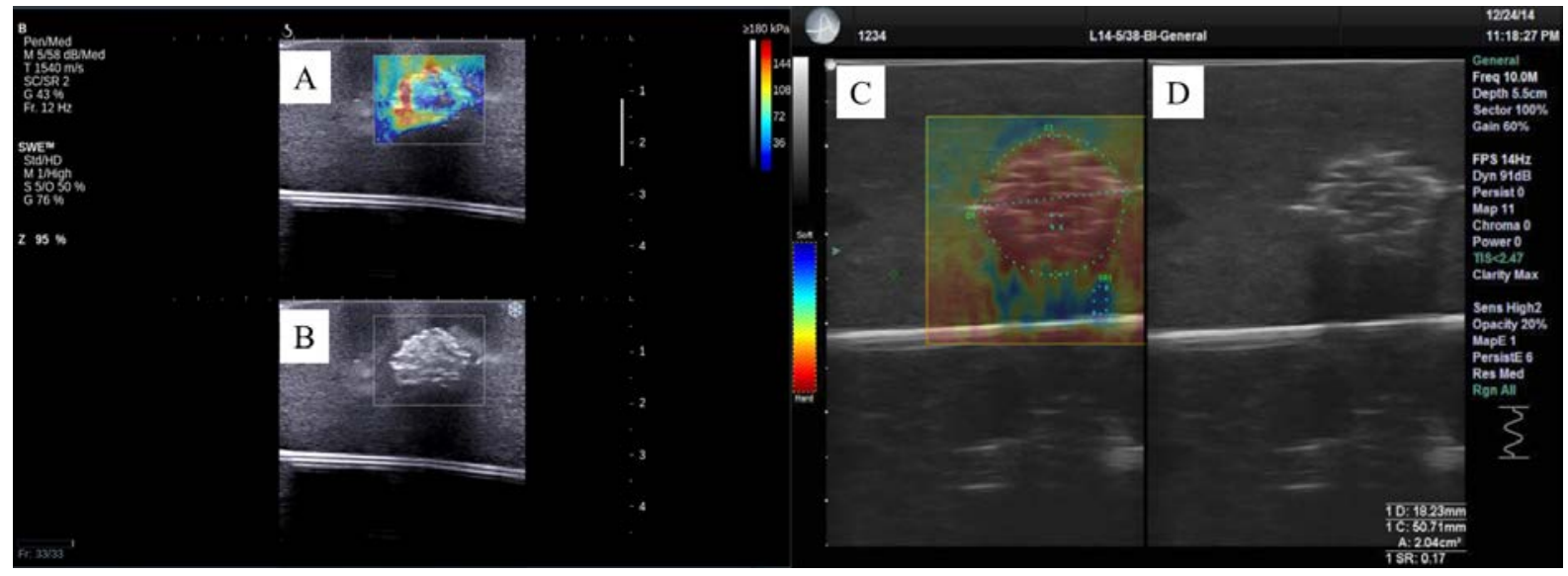

Figure 1: Figure A shows a tumor observed under SWE, B-mode ultrasound is shown in figure B and D. Figure $\mathrm{C}$ shows a tumor detected via $\mathrm{SE}$.

In figure 1A SWE image for the tumor demonstrates that the left periphery of the tumor is stiffer than the right periphery. This tumor is expected to be soft in the middle while on the periphery as a whole the tumor is stiff. However contradicting results are visible in figure $1 \mathrm{C}$ where SE image shows a uniform high stiffness in the tumor. Although the periphery is less stiff than the tumor itself but a spread of tumor is suspected in the surrounding tissue.

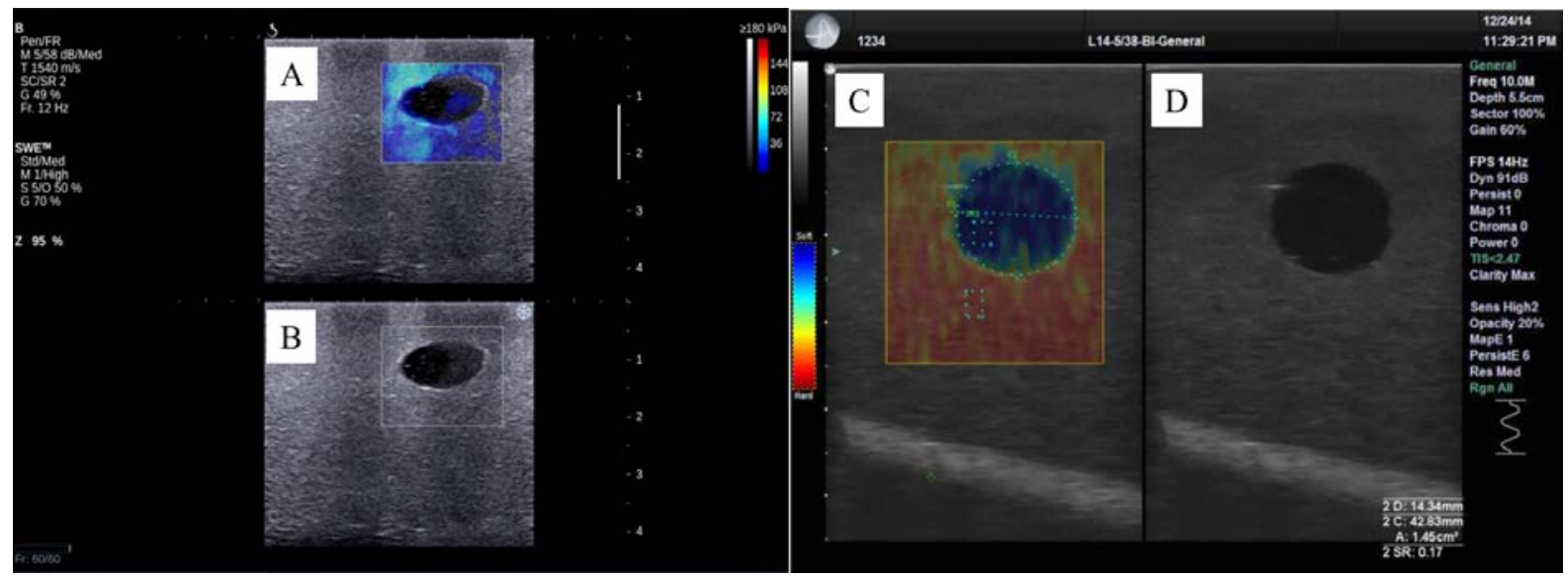

Figure 2: Figure A shows a cyst observed under SWE, B-mode ultrasound is shown in figure B and D. Figure C shows a cyst detected via SE.

Figure $2 \mathrm{~A}$ displays a cavity with elastographic analysis. The blue area indicates that there is no stiff tissue while the uncolored black area is the cavity. However in figure $2 \mathrm{C}$ a cavity is shown in blue color while the surrounding tissue displays a stiff tissue in comparison to the cavity itself. 
Mahdi Al-Qahtani, Eraj Humayun Mirza, Mubarak Dhafer Al-Qahtani and Mohammed Fahad Al-Muqati; A Comparative Study of

Shear-Wave Elastography and Strain Elastography on a Breast Phantom for Diagnosis of Tumor and Cyst. Journal of Biomedical Engineering and Medical Imaging, Volume 2, No 3, June (2015) , pp 24-28

In our study, SWE and SE showed similar results. Both elastographic modalities improved diagnostic performance in combination with normal B-mode ultrasound. However, sensitivity was improved in SWE. These results are similar to that of Chang et al. [11], where they compared SWE and SE on benign and malignant breast lesions. Our results for cyst is the first of its kind. The cyst shows a normal tissue without any significant stiffness for SWE imaging, however a difference in tissue stiffness can be observed for surrounding tissue under SE imaging. We suggest imaging of cyst is definitely better under SE imaging as it might provide detailed strain ratios in case where there is inflammation of surrounding tissue. Whereas, in case of SWE, if there is a difference in tissue stiffness for surrounding tissue it might not be detected as SWE imaging shows the cyst as a cavity while its surrounding tissue is displayed as normal.

\section{Conclusion}

Both elastography techniques (SWE \& SE) demonstrates promising future for diagnosis of breast tumours. However it is suggested that SE must be used for reviewing the cyst as it will provide more detailed diagnostic information for the surrounding tissue. Furthermore, Elastography must be combined with Bmode ultrasound to enhance the diagnostic performance.

\section{ACKNOWLEDGEMENT}

The authors extend their appreciation to the College of Applied Medical Sciences Research Center and the Deanship of Scientific Research at King Saud University for funding this research.

\section{REFERENCES}

[1] Krouskop, T.A., et al., Elastic moduli of breast and prostate tissues under compression. Ultrasonic imaging, 1998. 20(4): p. 260-274.

[2] Ophir, J., et al., Elastography: a quantitative method for imaging the elasticity of biological tissues. Ultrasonic imaging, 1991. 13(2): p. 111-134.

[3] Manning, F.A., L.M. Hill, and L.D. Platt, Qualitative amniotic fluid volume determination by ultrasound: antepartum detection of intrauterine growth retardation. American journal of obstetrics and gynecology, 1981. 139(3): p. 254-258.

[4] Ophir, J., et al., Elastography: ultrasonic estimation and imaging of the elastic properties of tissues. Proc Inst Mech Eng H, 1999. 213(3): p. 203-33.

[5] Bamber, J., et al., EFSUMB guidelines and recommendations on the clinical use of ultrasound elastographypart 1: Basic principles and technology. Ultraschall in der Medizin, 2013. 34(2): p. 169-184.

[6] Cortes, D.H., et al., Continuous Shear Wave Elastography: A New Method to Measure Viscoelastic Properties of Tendons in Vivo. Ultrasound Med Biol, 2015. 
[7] Athanasiou, A., et al., Feasibility of Imaging and Treatment Monitoring of Breast Lesions with Three-Dimensional Shear Wave Elastography. Ultraschall Med, 2015.

[8] Vural, M., et al., The evaluation of the retrobulbar orbital fat tissue and optic nerve with strain ratio elastography. Med Ultrason, 2015. 17(1): p. 45-8.

[9] Park, H.J., et al., Strain elastography features of epidermoid tumors in superficial soft tissue: Differences from other benign soft tissue tumors and malignant tumors. Br J Radiol, 2015: p. 20140797.

[10] Itoh, A., et al., Breast Disease: Clinical Application of US Elastography for Diagnosis 1. Radiology, 2006. 239(2): p. 341-350.

[11] Chang, J.M., et al., Comparison of shear-wave and strain ultrasound elastography in the differentiation of benign and malignant breast lesions. AJR Am J Roentgenol, 2013. 201(2): p. W347-56. 